\title{
Late heading of perennial ryegrass caused by introducing an Arabidopsis homeobox gene
}

P. van der Valk ${ }^{1,4}$, M. C. G. Proveniers ${ }^{2}$, J. H. Pertijs ${ }^{1}$, J. T. W. H. Lamers ${ }^{1}$, C. M. P. van Dun ${ }^{1,3}$ and J. C. M. SMEEKENS ${ }^{2}$

${ }^{1}$ Advanta Seeds B.V., Biotechnology Department, P.O. Box 1, 4410 AA Rilland, The Netherlands; ${ }^{2}$ Department of Molecular Plant Physiology, University of Utrecht, Padualaan 8, $3584 \mathrm{CH}$ Utrecht, The Netherlands; ${ }^{3}$ Present address: Rijk Zwaan Zaadteelt en Zaadhandel BV, Eerste Kruisweg 9, 4793 RS Fijnaart, The Netherlands; ${ }^{4}$ Present address: Vluchtenborch 10, 4411 DL Rilland, The Netherlands; E-mail: Pvandervalk@zeelandnet.nl

With 4 figures and 1 table

Received January 12, 2004/Accepted May 21, 2004

Communicated by T. Börner

\begin{abstract}
Perennial ryegrass (Lolium perenne L.) is the most important temperate forage grass species. Unfortunately, the nutritional value of perennial ryegrass declines as maturity progresses, mainly because of a high concentration of poorly digestible compounds in inflorescences. Therefore, the development of forage-type ryegrass varieties with extended vegetative growth is of interest for agriculture. To delay floral transition in perennial ryegrass the Arabidopsis ATH1 gene driven by the maize ubiquitin promoter, the rice actin promoter or the rice OSH1 promoter, respectively was introduced. In ATH1-expressing plants heading was delayed, and in a number of cases the plants never flowered at all. Such non- or late-heading was accompanied by the outgrowth of normally quiescent lateral meristems into extra leaves, resulting in a leafy growth habit. When eventually heading, these plants generally produced a reduced number of inflorescences. These observations suggest that $A T H 1$-mediated delay of heading may be useful to improve fodder quality of perennial ryegrass.
\end{abstract}

Key words: Lolium perenne - Arabidopsis homeobox gene biotechnology - late heading - ATH1 - transgenic grass

Ryegrasses are the most widely grown temperate grasses in the world. In addition to their native environment (Europe, North Africa and temperate Asia), they are distributed over North and South America, New Zealand and Australia. Ryegrasses are grown primarily for pasture, silage and hay. In recent years, their use for turf has also increased because of the selection of more dense-growing and persistent types (Hall 1992, Hannaway et al. 1999). Because of its high levels of palatability, digestible energy, protein and minerals, perennial ryegrass is considered the premier-quality forage grass throughout the world, and these properties make it suitable for all classes of livestock (Hannaway et al. 1999). The value of grass as forage, however, depends largely on the developmental stage of the plant. As maturity progresses a significant decrease in nutritional composition can be observed. In heading plants, crude protein levels, total digestible nutrients, and phosphorus decrease to $55 \%, 75 \%$ and $67 \%$, respectively, when compared with early vegetative plants, whereas digestible energy, metabolizable energy, and calcium levels decrease to about $85 \%$ (Hannaway et al. 1999). This is largely caused by high amounts of low digestible compounds in floral stems, like lignins and cell wall compounds coupled to lignin. Therefore, the development of ryegrass varieties with an extended vegetative growth phase or even varieties that remain vegetative is of interest.

The life cycle of perennial ryegrass consists of three principal developmental phases: a vegetative phase, a transition phase and a reproductive phase. The vegetative phase is characterized by meristems that form leaves. It starts at germination and continues through a process called tillering. Tillers are new grass shoots, consisting of an apical meristem, a stem, leaves, root nodes and latent buds. During the transition phase the apical meristem is gradually transformed from a vegetative to a floral bud. This transition to flowering depends upon the competence to respond to winter conditions with cold (vernalization) and short days followed by spring conditions with increased temperature and long days (LD). The conversion of the vegetative shoot apex into a floral bud is completed during the reproductive phase, resulting in the formation of an inflorescence.

In the closely related annual ryegrass, Lolium temulentum, photoperiodic induction of flowering can be induced by a single LD and is known to result from two sequential signals acting at the shoot apex. The first, still unidentified, signal induces the apex into a florally determined state and then the second signal, most likely the leaf-derived gibberellins $\mathrm{GA}_{5}$ and $\mathrm{GA}_{6}$, elicits expression of this state (McDaniel and Hartnett 1996, King et al. 2003, King and Evans 2003). An increase of $\mathrm{GA}_{1}$ and $\mathrm{GA}_{4}$ levels in both leaves and apices later in development, which occurs after exposure to multiple LDs, indicates a second GA action involved in inflorescence differentiation (Gocal et al. 2001b, King et al. 2003). Apart from a correlated up-regulation of $L t G A M Y B$ gene expression at the shoot apex, not much is known about the molecular mechanisms controlling ryegrass floral meristem identity (Gocal et al. 1999, 2001a,b; for review see King and Evans 2003).

In contrast, knowledge of the molecular processes underlying floral transition in Arabidopsis thaliana is much more comprehensive, and several key control genes in this process like LEAFY (LFY), TERMINAL FLOWER 1 (TFL1), APETALAI (API), CONSTANS (CO) and FLOWERING LOCUS T (FT) have been isolated (reviewed by Araki 2001, Mouradov et al. 2002). Over the past few years homologues of these genes have been identified and characterized in Lolium and other monocot species, indicating that there is a similarity 
between the processes controlling flowering in dicots and monocots (Gocal et al. 2001a, Jensen et al. 2001, Bomblies et al. 2003, Hayama et al. 2003). This is supported by the fact that constitutive overexpression of the dicot Arabidopsis LFY gene in the monocot rice causes early heading, similar to its function in Arabidopsis (He et al. 2000). Conversely, expression of the Lolium TFL1 and API homologues in the Arabidopsis $t f 1$ and apl mutants, respectively, show that these genes can (partially) substitute for their Arabidopsis counterparts (Gocal et al. 2001a, Jensen et al. 2001). In addition, a $\mathrm{CO}$-like sequence was found to map close to the major quantitative trait locus for heading date in Lolium (Donnison et al. 2002).

For this reason, it was decided to constitutively express the Arabidopsis TALE-homeobox gene $A T H 1$ in perennial ryegrass to delay or prevent the transition to flowering (Quaedvlieg et al. 1995, Bürglin 1997). ATH1 was first identified in a screen for light-regulated transcription factor genes in Arabidopsis. In Arabidopsis seedlings ATH1 is highly expressed in the shoot apical meristem (SAM) and leaf primordia (Quaedvlieg et al. 1995). Prior to floral transition, ATH1 SAM expression is gradually down-regulated to undetectable levels. Arabidopsis plants constitutively expressing (anti)sense $A T H 1$ and athl mutants display a flowering time phenotype that suggests that ATH1 functions as an inhibitor of floral transition (M. C. G. Proveniers and J. C. M. Smeekens, unpublished results). In tobacco, constitutive expression of ATH1 results in late flowering as a consequence of impaired GA biosynthesis (M. C. G. Proveniers, B. P. W. Rutjens, C. M. Brand and J. C. M. Smeekens, unpublished results). Here, it is shown that expression of ATH1 in L. perenne, under control of the maize ubiquitin (Ubi) promoter, the rice actin (Act) promoter or the rice OSH1 promoter not only results in significant later heading, but also in increased formation of leaves.

\section{Materials and Methods}

Plant material, tissue culture, growth conditions and flowering experiments: Seeds of the varieties 'Mondial' and 'Kelvin' of the outbreeding grass species L. perenne L. were used for the production of embryogenic callus as described by Spangenberg et al. (1995). Extensive screening of a large number of seeds resulted in the identification of a few highly embryogenic genotypes. Several clones of these elite genotypes were maintained in the greenhouse. Embryogenic suspension cultures were established from callus induced on immature inflorescences from vernalized plants of a single elite genotype of the variety 'Mondial'.

For flowering experiments, both transformed and non-transformed control plants of the single elite genotype (var. 'Mondial', about 10 tillers/plant) were vernalized in a growth chamber for 70 days $\left(5^{\circ} \mathrm{C}\right.$; $8 \mathrm{~h}$ light, $16 \mathrm{~h}$ dark). After vernalization plants were transferred to LD conditions $\left[16 \mathrm{~h}\right.$ light $\left(12^{\circ} \mathrm{C}\right), 8 \mathrm{~h}$ dark $\left.\left(8^{\circ} \mathrm{C}\right)\right]$ in a greenhouse with supplementary light from sodium and mercury sources $(100 \mu \mathrm{mol}$ photons $/ \mathrm{m}^{2} / \mathrm{s}$ ). After 1-2 weeks the day and night temperatures were raised by $6^{\circ} \mathrm{C}$. Plants were scored weekly for the presence of inflorescences. A plant was scored as 'heading' on the day the first inflorescence became visible.

Genetic transformation: Embryogenic suspension cultures were transformed by particle bombardment using the Particle Inflow Gun (Finer et al. 1992). Three plasmids (pVDH636, pVDH743, pVDH728) containing the ATH1 cDNA were used for transformations. These plasmids contained the hygromycin phosphotransferase marker gene driven by the rice Act promoter (Spangenberg et al. 1995). The following promoters were used to drive $A T H 1$ gene expression: (1) the maize Ubi-promoter (Christensen et al. 1992), (2) the rice Actpromoter (McElroy et al. 1991), and (3) the rice OSH1-promoter (Matsuoka et al. 1993). Promoter-GUS fusions were made to localize the activity of the selected promoters in perennial ryegrass. Hygromycin selection of transformants was as described by Spangenberg et al. (1995). Transgene presence in primary transformants was confirmed by polymerase chain reaction (PCR) analysis.

Gene expression assay: $A T H 1$-expression in young leaf material (up to $10 \mathrm{~cm}$ leaf length) was assayed using quantitative real time PCR (RT-PCR) (Taqman ${ }^{\circledR}$ procedure, ABI7700 detection system; Applied Biosystems, Foster City, CA, USA). A L. perenne homologue of the rice Act 7 gene (GenBank accession number X15863) was used as an internal expression control. Primers and probes for both the ATH1 gene and the expression control gene were developed using Primer express $^{\mathrm{TM}}$ (version 1.0) according to the manufacturer's instructions (Applied Biosystems).

Total RNA was isolated from six to eight leaf tips $(1 \mathrm{~cm})$ using RNeasy mini spin columns (Qiagen, Hilden, Germany). RT-reaction was carried out using the Thermoscript ${ }^{\mathrm{TM}}$ RNase H-Reverse Transcriptase Kit (Invitrogen, San Diego, CA, USA).

Quantitative analysis of GA derivatives: For quantitative analysis of GAs young leaves (up to $10 \mathrm{~cm}$ length) were harvested under LD conditions at a fixed time of the day. Ground tissue was lyophilized, weighed and extracted in cold $\left(4^{\circ} \mathrm{C}\right)$ methanol : water $(4: 1, \mathrm{v} / \mathrm{v})$ $(50 \mathrm{ml})$. GA purification and analysis were performed as described by Coles et al. (1999).

\section{Results}

Since ATH1 in Arabidopsis is predominantly expressed at the site of floral transition, the vegetative shoot apex (Quaedvlieg et al. 1995; M. C. G. Proveniers and J. C. M. Smeekens, unpublished data), three different monocot promoters fused to the GUS marker gene were tested for their ability to induce

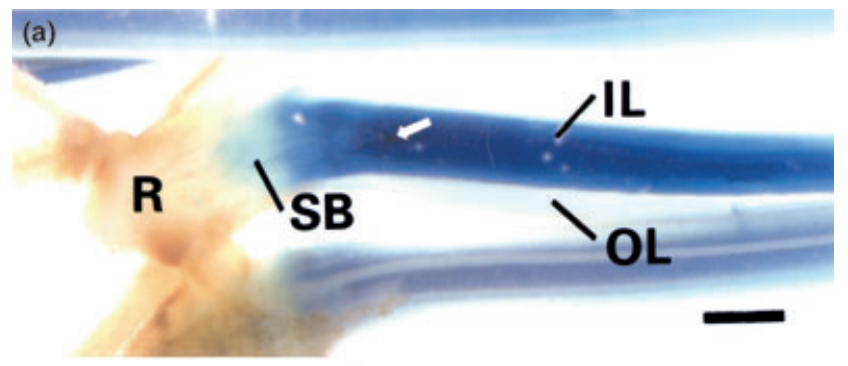

(b)

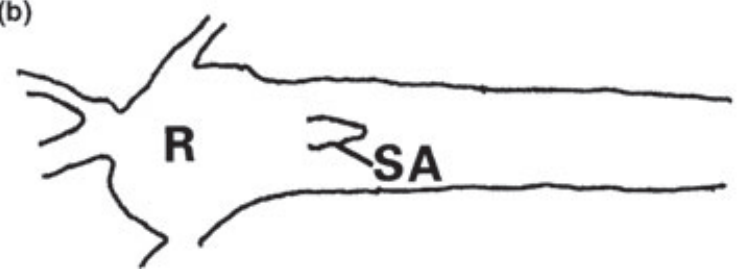

Fig. 1: (a) Longitudinal section through GUS-stained ubiquitin (Ubi)::GUS-transgenic Lolium perenne plantlet (print from slide). Note heavy staining at the site of the shoot apex (arrow) (see: Fig. 1b) and in the surrounding young (inner) leaves (IL). Older (outer) leaves (OL) and roots $(\mathrm{R})$ remain unstained. Because of the lack of contrast between shoot apex and surrounding inner leaves, the shoot apex does not stand out. Therefore, a schematic drawing (directly from slide) of the GUS-stained plantlet of Fig. 1a is presented (b). SA, shoot apex; $\mathrm{SB}$, shoot base. Scale bar $=1 \mathrm{~mm}$ 
high levels of expression in corresponding tissues of L. perenne. Histological examination of GUS-positive transgenic Lolium plants showed that for all three promoters tested (maize Ubi, rice Act, and rice OSH1) heavy staining occurred at the site of the shoot apex and in young leaves (Fig. 1). Staining was somewhat stronger using the Ubi- and the Act-promoter when compared with the OSH1-promoter. Older leaves and roots remained unstained (Fig. 1).

To investigate whether $A T H 1$ overexpression in ryegrass affects floral transition as it does in Arabidopsis and tobacco plants, embryogenic suspension cultures of $L$. perenne var. 'Mondial' were transformed with ATH1 cDNA (Quaedvlieg et al. 1995) fused to each one of these promoters. For the Ubi::ATH1 construct a total number of 59 independent regenerants were obtained. Fifty-one of these were found to contain the transgene, whereas eight plants appeared to be nontransformed. Ten clones of each regenerant and of a nontransformed control were subjected to a flowering experiment. Compared with non-transformed control plants transgenecontaining plants showed, on average, a delay in heading (37.3 days vs. 29.9 days after the first LD) (Table 1, Expt A). It

Table 1: Mean heading times (HT) in days for ubiquitin (Ubi)::ATH1transformants (T) and non-transformed (NT) controls

\begin{tabular}{lc}
\hline Group & Heading time (HT) \\
\hline Experiment A & \\
T, PCR $(+)$ & $37.3^{\mathrm{b}}$ \\
T, PCR $(-)$ & $33.6^{\mathrm{a}}$ \\
NT & $29.9^{\mathrm{a}}$ \\
Experiment B & $48.7^{\mathrm{b}}$ \\
T, PCR $(+)$, transgene expressing & $32.4^{\mathrm{a}}$ \\
NT & \\
\hline
\end{tabular}

Different letters indicate significant differences at $\mathrm{P}=0.05$. $\mathrm{PCR}(+)$ : transgene containing transformants, consisting of both transgene expressing and non-expressing plants; $\mathrm{PCR}(-)$ : no-transgene containing transformants; NT: non-transformed control plants. Heading time [appearance of first inflorescence expressed as number of days after first long day (LD)] was scored weekly at the same day of the week. Scoring was continued until 85 days after the first LD. Transgene expression was measured by quantitative real time polymerase chain reaction (RT-PCR). is known that in Lolium and other monocot species, tissue culture and particle bombardment can cause late heading because of chromosomal changes (Stadelmann et al. 1998, Choi et al. 2000a,b). Indeed, late heading was also observed in some of the transformants lacking the transgene (Table 1, Expt A) or lacking transgene expression (data not shown). However, when compared with both the group of non-transformed control plants and the group of transformed plants lacking the transgene, heading in the group of transgene-containing transformants was significantly later (Table 1, Expt A), indicating that this lateness can be attributed to transgene expression.

Quantitative RT-PCR analysis revealed that 37 of the 51 transgene-containing lines showed expression of the transgene, with expression levels per line ranging from high to low. On average, the group of $A T H 1$ expressing lines headed 16 days later than the group of control plants (Table 1, Expt B). Moreover, a substantial number of plants failed to show heading within the time frame of the experiment ( 84 days after the first LD) (Fig. 2b). Non-transformed control plants formed numerous $(>10)$ inflorescences and had an open structure (Fig. 2a, right). Interestingly, most late- and nonheading $A T H 1$-expressing plants were very leafy with a compact appearance because of an increased outgrowth of lateral meristems into leaves [Fig. 2a (left),b]. Normally, such outgrowth of lateral meristems is inhibited. In a number of non-heading transgenics a more open growth habit was observed, caused by the formation of branched, elongated, raised, vegetative tillers that had originated through internode extension (Fig. 2c,d) (Minderhoud 1978, 1980). This form of tillering is well-known in L. perenne and is often associated with a disturbed apical growth (Krijne 1958).

When eventually heading, $A T H 1$-expressing plants generally produced a reduced number of inflorescences. Inflorescence development further proceeded without abnormalities and normal viable pollen was produced (lacto-fuchsin staining; data not shown).

For a second, large-scale flowering experiment 288 plants of each of four different lines were used: one non-transformed control line and three expressing Ubi::ATH1 transgenic lines with different mean heading times as determined from the first
Fig. 2: Late-heading $A T H 1$-expressing perennial ryegrass with characteristic compact, leafy growth. (a) Non-heading ubiquitin (Ubi):: ATH1 plant (left) and heading non-transformed control with numerous inflorescences (right) both 6 weeks after first long day (LD); (b) non-heading Ubi::ATH1 plant 9 weeks after first LD; (c) OSH1::ATH1 plant with elongated, raised, vegetative tillers; (d) branched vegetative tiller of OSH1::ATH1 plant originated through intravaginal tillering with internode extension
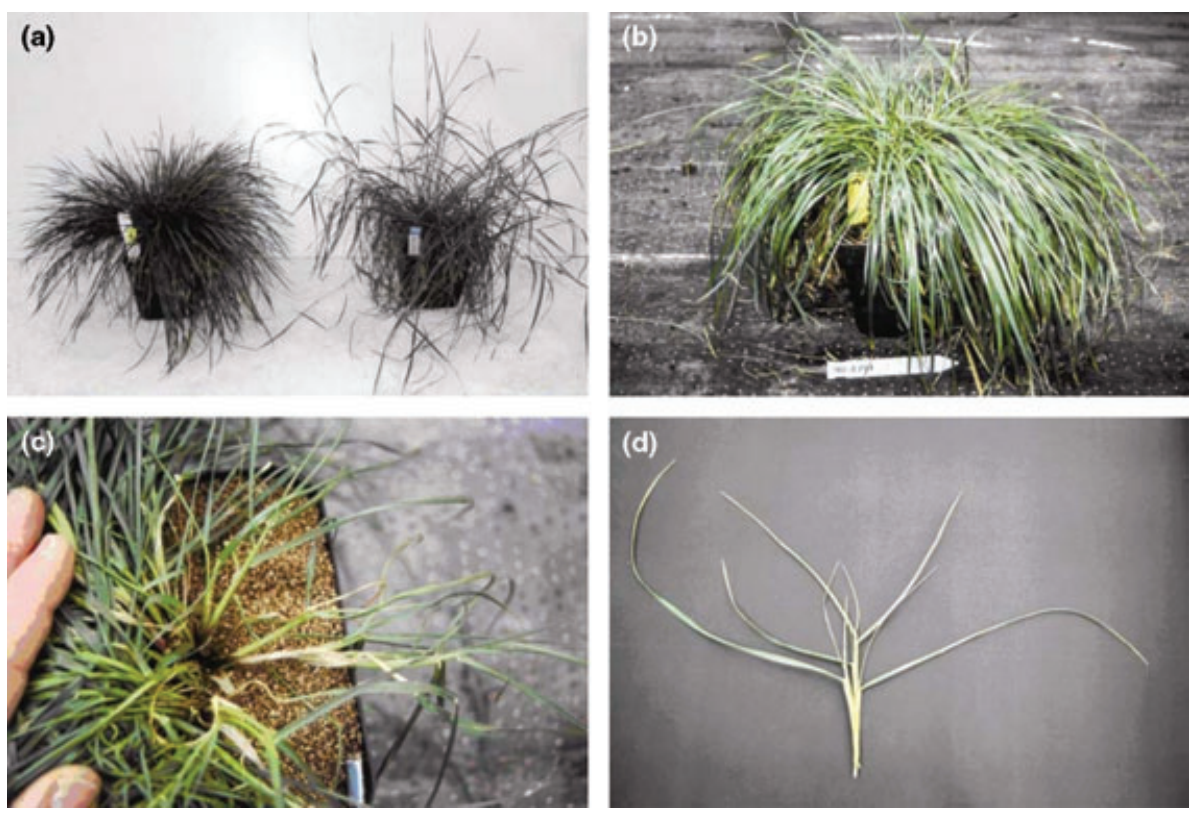


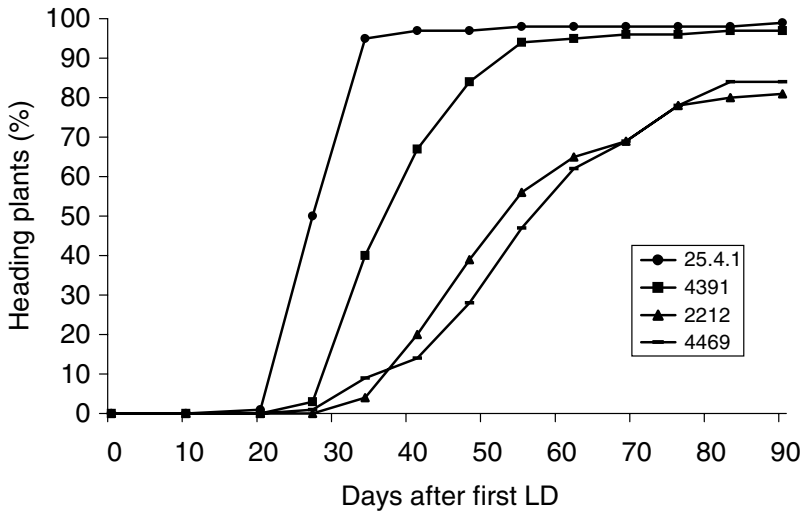

Fig. 3: Cumulative percentages of heading of ubiquitin (Ubi)::ATH1 and untransformed control plants over a period of 90 days after first long day $(\mathrm{LD})(100 \%=288$ plants $)$. Line $25.4 .1=$ non-transformed control; lines 2212, 4391 and 4469 are Ubi::ATH1-transgenics

experiment. Figure 3 shows that virtually all non-transformed control plants (line 25.4.1) headed within a 10-day interval, between 20 and 30 days after the first LD, whereas the three transgenic lines showed various degrees of delay of heading. Two of the lines (2212 and 4469) reached 50\% heading over 1 month later than the non-transformed controls, confirming the results of the first trial. As in the previous trial, these lines never reached $100 \%$ heading. After 3 months, at the end of the experiment, about $20 \%$ of the plants from both lines were still in the vegetative growth phase (Figs 3 and 4). These nonheading plants were healthy-looking as were heading plants of the same line, excluding the possibility that a plant disease was the cause of non-heading. As observed before, in most cases late heading was accompanied by a reduction in the number of inflorescences formed. Whereas the majority of control plants $(>90 \%)$ always flowered with more than 10 inflorescences [Fig. 2a (right); Fig. 4], a considerable number of late-heading

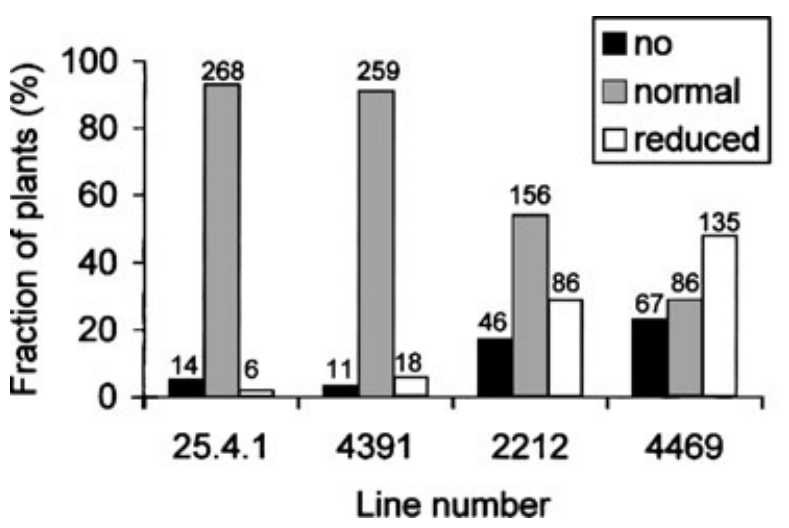

Fig. 4: Fraction of plants (\%) per inflorescence class (no inflorescences, normal or reduced number of inflorescences) in ubiquitin (Ubi)::ATH1 and untransformed control plants 11 weeks after first long day (LD) $(100 \%=288$ plants). At this point in time the number of heading control plants was at its maximum (95\%) (Fig. 3). Normalheading plants always headed with $>10$ inflorescences. Line $25.4 .1=$ non-transformed control; lines 2212, 4391 and 4469 are Ubi::ATH1transgenics. For the 'reduced' class the average number of inflorescences was as follows: line $25.4 .1=2.0$; line $4391=5.8$; line $2212=$ 4.9 ; line $4469=3.6$. Figures above columns represent total number of plants per inflorescence class per line. Total number of plants per line $=288$ plants (up to $70 \%$ of the plants of line 4469) formed substantially fewer inflorescences, ranging from 3.6 to 5.8 inflorescences per plant (Fig. 4). Figure 3 further shows that transgenic lines showing delayed heading also showed increased variation in heading times when compared with the non-transformed control.

Similar flowering time experiments were performed with Act::ATH1- and OSH1::ATH1-transformants. Like Ubi::ATH1 plants, Act::ATH1 and OSH1::ATH1 plants were late heading and displayed a leafy, vegetative phenotype.

In tobacco, late flowering of $A T H 1$ overexpressing plants coincides with significantly decreased $\mathrm{GA}_{1}$ levels and the phenotype can be corrected by application of bioactive GA to the plants (M. C. G. Proveniers, B. P. W. Rutjens, C. M. Brand and J. C. M. Smeekens, unpublished results). Gibberellins are also important for floral transition in Lolium, with $\mathrm{GA}_{1}$ functioning as a secondary, late-acting photoperiod-induced stimulus for inflorescence differentiation (King et al. 2001). It was, therefore, decided to see whether $A T H 1$ overexpression in L. perenne also affected GA levels. Indeed, initial results show that young leaf material from high $A T H 1$-expressing lines have two- to threefold decreased levels of $\mathrm{GA}_{1}(1.6 \mathrm{ng} / \mathrm{g} \mathrm{DW}$ in non-transformed controls vs. $0.5-0.7 \mathrm{ng} / \mathrm{g} \mathrm{DW}$ in overexpressor lines).

\section{Discussion}

To delay floral transition in perennial ryegrass, this grass species was genetically transformed, using particle bombardment with the Arabidopsis homeobox gene ATH1. Expression of this gene, driven by each of three different monocot promoters, maize Ubi, rice Act, and rice OSH1, caused delayed heading. In a number of transgenic lines plants failed to flower at all. Non- or late-heading plants usually were leafy, resulting from the outgrowth of normally quiescent lateral meristems. Late-heading plants generally produced a reduced number of inflorescences. Lines, showing delayed heading, also showed increased variation in heading times when compared with the non-transformed controls (Fig. 3). This increased variation in heading times may be caused by variation in transgene expression between vegetatively propagated clones of the same line. Variation in transgene expression has been observed between tillers of the same plant and between clones after vegetative propagation of Ubi::GUS-transgenic L. perenne (P. van der Valk, unpublished observations) and Act::GUS-transgenic Festuca arundinacea (Bettany et al. 1998).

In rice, reduced levels of $\mathrm{GA}_{1}$ caused by ectopic expression of OsGA2ox 1 cDNA have been found in close correlation with late flowering (Sakamoto et al. 2001). Similarly, the reduction in $\mathrm{GA}_{1}$ observed here might underlie the effect of ATH1 overexpression on heading time. However, future experiments have to show whether this is indeed the case, and whether other GAs are also changed. Candidates for further study are $\mathrm{GA}_{5}$ and $\mathrm{GA}_{6}$ as these intermediates play a role in early floral transition (King et al. 2001). Taken together, the effect of ectopic expression of ATHI in the monocot L. perenne is similar to that which has been observed in Arabidopsis and tobacco.

In perennial ryegrass the decrease in nutritional value associated with floral transition because of the formation of lignin-rich flower and stem material is significant. By contrast, biomass produced during the vegetative part of the life cycle mainly consists of easily digestible organic matter in the form 
of leaves. Late flowering varieties have improved fodder value because of their prolonged vegetative growth phase. In possible, future $A T H 1$-expressing ryegrass varieties additional quality improvement may be generated by the outgrowth of normally quiescent lateral meristems into extra leaves, and a reduced number of inflorescences.

Flowering pathways in monocots and dicots show some resemblance, but are not completely conserved as can be inferred from transformation experiments with Lolium homologues of Arabidopsis flowering time genes in Arabidopsis (Gocal et al. 2001a, Jensen et al. 2001). Therefore, a Lolium ATH1 homologue is being isolated to study its role in flowering control in ryegrass.

\section{Acknowledgements}

The authors thank Dr Peter Hedden and Steve Croker of the IACR, Long Ashton, UK, for use of their GA analysis facilities and assistance thereby. They also thank Mr Matthieu C. Défize for expert electronic artwork. This work was supported by the Dutch Ministry of Economic Affairs (BTS grant 99085).

\section{References}

Araki, T., 2001: Transition from vegetative to reproductive phase. Curr. Opin. Plant Biol. 4, 63-68.

Bettany, A. J. E., S. J. Dalton, E. Timms, and P. Morris, 1998: Stability of transgene expression during vegetative propagation of protoplast-derived tall fescue (Festuca arundinacea Schreb.) plants. J. Exp. Bot. 49, 1797-1804.

Bomblies, K., R. L. Wang, B. A. Ambrose, R. J. Schmidt, R. B. Meeley, and J. Doebley, 2003: Duplicate FLORICAULA/LEAFY homologs $z f l$ and $z f l 2$ control inflorescence architecture and flower patterning in maize. Development 130, 2385-2395.

Bürglin, T. R., 1997: Analysis of TALE superclass homeobox genes (MEIS, PBC, KNOX, Iroquois, TGIF) reveals a novel domain conserved between plants and animals. Nucleic Acids Res. 25, $4173-4180$.

Choi, H.-W., P. G. Lemaux, and M.-.J. Cho, 2000a: Increased chromosomal variation in transgenic versus nontransgenic barley (Hordeum vulgare L.) plants. Crop Sci. 40, 524 533.

Choi, H.-W., P. G. Lemaux, and M.-J. Cho, 2000b: High frequency of cytogenetic aberration in transgenic oat (Avena sativa L.) plants. Plant Sci. 156, 85-94.

Christensen, A. H., R. A. Sharrock, and P. H. Quail, 1992: Maize polyubiquitin genes: structure, thermal perturbation of expression and transcript splicing, and promoter activity following transfer to protoplasts by electroporation. Plant Mol. Biol. 18, 675-689.

Coles, J. P., A. L. Phillips, S. J. Croker, R. Garcia-Lepe, M. J. Lewis, and P. Hedden, 1999: Modification of gibberellin production and plant development in Arabidopsis by sense and antisense expression of gibberellin 20-oxidase genes. Plant J. 17, 547-556.

Donnison, I., P. Cisneros, T. Montoya, I. Armstead, B. Thomas, A. Thomas, N. Jones, and P. Morris, 2002: The floral transition in model and forage grasses. Flowering Newslett. 33, 42-48.

Finer, J. J., P. Vain, M. W. Jones, and M. D. McMullen, 1992: Development of the particle inflow gun for DNA delivery to plant cells. Plant Cell Rep. 11, 323-328.

Gocal, G. F. W., A. T. Poole, F. Gubler, R. J. Watts, C. Blundell, and R. W. King, 1999: Long-day up-regulation of a GAMYB gene during Lolium temulentum inflorescence formation. Plant Physiol. 119, $1271-1278$.

Gocal, G. F. W., R. W. King, C. A. Blundell, O. M. Schwartz, C. H. Andersen, and D. Weigel, 2001a: Evolution of floral meristem identity genes. Analysis of Lolium temulentum genes related to APETALA1 and LEAFY of Arabidopsis. Plant Physiol. 125, $1788-1801$.
Gocal, G. F. W., C. C. Sheldon, F. Gubler, T. Moritz, D. J. Bagnall, C. P. MacMillan, S. F. Li, R. W. Parish, E. S. Dennis, D. Weigel, and R. W. King, 2001b: GAMYB-like genes, flowering, and gibberellin signaling in Arabidopsis. Plant Physiol. 127, 1682-1693.

Hall, M. H., 1992: Ryegrass. Pennsylvania State University Coop. Extension Service Agronomical Facts 19.

Hannaway, D., S. Fransen, J. Cropper, M. Teel, M. Chaney, T. Griggs, R. Halse, J. Hart, P. Cheeke, D. Hansen, R. Klinger, and W. Lane, 1999: Perennial Ryegrass (Lolium perenne L.). Pacific Northwest Extension Publications, Oregon State University, (http://www.eesc. orst.edu/agcomwebfile/edmat $/ \mathrm{html} / \mathrm{pnw} / \mathrm{pnw} 503 / \mathrm{pnw} 503 . \mathrm{html})$.

Hayama, R., S. Yokoi, S. Tamaki, M. Yano, and K. Shimamoto, 2003: Adaptation of photoperiodic control pathways produces short-day flowering in rice. Nature 422, 719-722.

He, Z., Q. Zhu, T. Dabi, D. Li, D. Weigel, and C. Lamb, 2000: Transformation of rice with the Arabidopsis floral regulator $L E A F Y$ causes early heading. Transgenic Res. 9, 223-227.

Jensen, C. S., K. Salchert, and K. K. Nielsen, 2001: A TERMINAL FLOWERI-like gene from perennial ryegrass involved in floral transition and axillary meristem identity. Plant Physiol. 125, $1517-1528$.

King, R. W., and L. T. Evans, 2003: Gibberellins and flowering of grasses and cereals: prizing open the lid of the 'florigen' black box. Annu. Rev. Plant Biol. 54, 307-328.

King, R. W., T. Moritz, L. T. Evans, O. Junttila, and A. J. Herlt, 2001: Long-day induction of flowering in Lolium temulentum involves sequential increases in specific gibberellins at the shoot apex. Plant Physiol. 127, 624 632.

King, R. W., L. T. Evans, L. N. Mander, T. Moritz, R. P. Pharis, and B. Twitchin, 2003: Synthesis of gibberellin $\mathrm{GA}_{6}$ and its role in flowering of Lolium temulentum. Phytochemistry 62, 77-82.

Krijne, A. A., 1958: Een morfologisch onderzoek naar de vegetatieve groei van Engels raaigras (Lolium perenne L.). I.B.S. Mededeling 55, 99-105.

Matsuoka, M., H. Ichikawa, A. Saito, Y. Tada, T. Fujimura, and Y. Kano-Murakami, 1993: Expression of a rice homeobox gene causes altered morphology of transgenic plants. Plant Cell 5, 1039-1048.

McDaniel, C. N., and L. K. Hartnett, 1996: Flowering as metamorphosis: two sequential signals regulate floral initiation in Lolium temulentum. Development 122, 3661-3668.

McElroy, D., A. D. Blowers, B. Jenes, and R. Wu, 1991: Construction of expression vectors based on the rice actin $1($ Actl $) 5^{\prime}$ region for use in monocot transformation. Mol. Gen. Genet. 231, 150-160.

Minderhoud, J. W., 1978: Pseudostolons and aerial tillers: morphological phenomena of Lolium perenne L. Proceedings of the 7th General Meeting of the European Grassland Federation. Gent. (1978). Soc. for Grassland and Fodder Crops. pp. 10.31-10.39.

Minderhoud, J. W., 1980: Tillering and persistency in perennial ryegrass. Proceedings of the Third International Turfgrass Research Conference. Munich 1977, ASA-CSSA-SSSA and Int. Turfgrass Soc., pp. 97-107.

Mouradov, A., F. Cremer, and G. Coupland, 2002: Control of flowering time. Interacting pathways as a basis for diversity. Plant Cell 14, Supplement, S111-S130.

Quaedvlieg, N., J. Dockx, F. Rook, P. Weisbeek, and S. Smeekens, 1995: The homeobox gene ATH1 of Arabidopsis is derepressed in the photomorphogenic mutants copl and det1. Plant Cell 7, 117-129.

Sakamoto, T., M. Kobayashi, H. Itoh, A. Tagiri, T. Kayano, H. Tanaka, S. Iwahori, and M. Matsuoka, 2001: Expression of a gibberellin 2-oxidase gene around the shoot apex is related to phase transition in rice. Plant Physiol. 125, 1508-1516.

Spangenberg, G., Z.-Y. Wang, X. Wu, J. Nagel, and I. Potrykus, 1995: Transgenic perennial ryegrass (Lolium perenne) plants from microprojectile bombardment of embryogenic suspension cells. Plant Sci. 108, 209-217.

Stadelmann, F. J., B. Boller, G. Spangenberg, R. Kölliker, Z.-Y. Wang, I. Potrykus, and J. Nösberger, 1998: Field performance of cellsuspension derived Lolium perenne L. regenerants and their progenies. Theor. Appl. Genet. 96, 634 639 . 